\title{
Extraction and Characterization of Proanthocyanidins from Grape Seeds
}

\author{
Sean X. Liu*,1 and Elizabeth White ${ }^{2}$ \\ ${ }^{1}$ Functional Foods Research Unit, National Center for Agricultural Utilization Research USDA, ARS 1815 N. University \\ Street, Peoria, IL 61604, USA \\ ${ }^{2}$ Food Science Department, Rutgers University, 65 Dudley Road, New Brunswick, NJ 08901, USA
}

\begin{abstract}
Grape seed contains many polyphenolic compounds that have potential health-promoting benefits. Among these compounds are proanthocyanidins, also known as condensed tannins, which is a class of flavanols. The basic structural building blocks of flavanols are polyhydroxyflavan-3-ol units linked together by C-C bonds. They are widely distributed in plants but grape seed has very high contents. This study examined extraction and purification technologies for obtaining proanthocyanidins from grape seeds and analytical methods and tools for characterizing them.
\end{abstract}

Keywords: Grape seeds, proanthocyanidins, phenolic compounds, Polyphenols, HPLC, extraction, bioactive compounds.

\section{INTRODUCTION}

Polyphenolic compounds are common in all plants. They are necessary components of plant physiology because of their involvement in the areas of plant color, growth, increased resistance to predation due to astringency of taste, and many other factors [1]. While polyphenolic compounds do vary from species to species of different plants, and in some cases even between variety of plants within a species, their potential to be used as healthful compounds for improving human health, when they are consumed, is the same.

Proanthocyanidins are polyphenolic compounds that can be found in the plant physiology of several plant species, are mainly concentrated in tree barks and outer skins of seeds. Grape seeds are one of the richest sources of proanthocyanidins [2]. Public awareness of the antioxidant and free radical-scavenging abilities of these phenolic compounds results largely from the potential of these compounds to increase human health in many ways. Reductions in cardiovascular disease, cerebrovascular diseases, and cancer mortality are just some of the noticeable examples associated with these phenolic compounds [3-5]. Recent advances in the field of nutraceuticals have shown that the compounds found in grapes exhibit even more benefits than initially believed. This paper aims at reviewing these compounds found in the anatomy of the grape seeds and current methods of separation and characterization of proanthocyandins.

\section{OVERVIEW OF SEPARATION METHODS}

\subsection{Chemistry of Proanthocyanidins}

Proanthocyanidins are commonly referred to as condensed tannins and a class of flavanols, which belong to the larger group of polyphenolic compounds. Proanthocyanidins

*Address correspondence to this author at the Functional Foods Research Unit, National Center for Agricultural Utilization Research USDA ARS, 1815 N. University Street, Peoria, IL 61604, USA; Tel: 1-309-681-6551; Fax: 1-309-681-6685; E-mail: sean.liu@ars.usda.gov can be viewed as essentially polymer chains of flavanols such as catechins. Polyphenolic compounds evolve from the plant's natural metabolic synthesizing pathways of either the acetate pathway or the shikimate pathway [1]. Polyphenolic compounds come in a number of chemical structures and sizes consisting of different monomer units. There is great diversity amongst the monomer units and basic structures of the compounds produced. Polyphenols can exist in monomer form, however they are usually found in conjugated form in nature. Sugars are the most common compounds to attach to polyphenols; these compounds will also form bonds with other phenol groups, various organic acids, and even lipids. It is the hydroxyl group of the polyphenolic compound, more often than not, which links to sugar residues found naturally within the plant. These sugar compounds vary in chain length size and composition, though glucose is the most often found due to its prevalence in plant physiology. While it is possible for plant sugar residue to link to one of the aromatic carbons of the compound, this is not the most common form of attachment.

In 1989, Harborne categorized the major plant producing polyphenolic compounds into classes [1]. This classification was primarily designed based on the chemical structure of the polyphenol. The compounds can be seen varying in size based on carbon skeleton structure and also on the side chains associated with them. With respect to grape seeds and the proanthocyanidins associated with them, the most important group on the chemical structure is the flavonoids grouping. Harborne further separated this polyphenolic group into 13 subcatagories, with proanthocyanidins being one of the classes [6].

Proanthocyanidins are of a diphenylpropane structure of $\mathrm{C}_{6}$ $\mathrm{C}_{3}-\mathrm{C}_{6}$. Most often found as a glycoside derivative, this compound class is composed of three monomer units of catechin, epicatechin, and epigallocatechin [7] (See Fig. 1). Proanthocyanidins vary in size from monomer form to oligomer form, occurring as monomers, dimers, trimers, and oligomers as large as 20 or more units in the form, though higher molecular weight polymers are typically the norm [3]. 


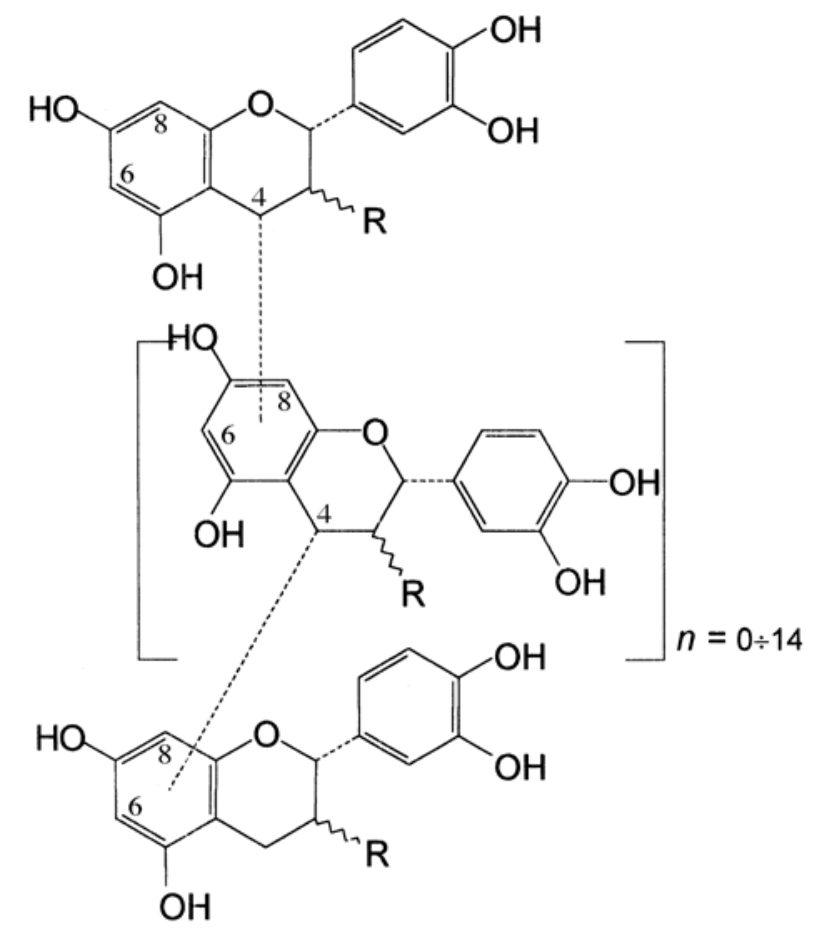

$\mathrm{R}=3 \beta-\mathrm{OH} \quad$ monomer: catechin

$\mathrm{R}=3 \alpha-\mathrm{OH} \quad$ monomer: epicatechin

$\mathrm{R}=3 \beta-O$-gallate $\quad$ monomer: catechin 3-O-gallate

$\mathrm{R}=3 \alpha-O$-gallate $\quad$ monomer: epicatechin 3-O-gallate

Fig. (1). Several possible building blocks of proanthocyandins from.

The various forms of these compounds allows for most of them to be water soluble, though larger condensed tannins have been found to be insoluble.

\subsection{Healthful Properties of Proanthocyanidins}

Proanthocyanidins, as a whole, cause many bioactivities that produce positive, healthful changes in the human body. It had been demonstrated that these compounds exhibit antioxidant properties and help the body ward off cardiovascular disease $[4,8,9]$, various immune disorders, and neurodegenerative disease [10-12]. Perhaps the most widely known health related anecdote from products associated with grapes was the explanation of so-called "French paradox", in which red wine was shown to reduce the risk of coronary heart disease $[13,14]$. However, all phenolic compounds with antioxidant properties react in the same manner with free radicals to help prevent all of the above afflictions. Total phenols (or antioxidant effect) of a plant species can be measured using the Folin-Ciocalteu reaction. Results are typically expressed as gallic acid equivalents (GAE).

Phenolic compounds as a whole are healthful due to both their ability to terminate free radicals and to acting as metal chelators. Both free radicals and metals are known to help catalyze lipid peroxidation reactions, which leads to many human ailments. The proanthocyanidins and other healthful phenolic compounds prevent this and other oxidation reactions via hydrogen donation from the phenol to the free radical containing compound. The typical base structure with ring stability helps support the free radical that it is left with and therefore does not react again to initiate oxidation. (See Reactions A) However, the free radical polyphenol can also act as a terminator for a free radical reaction sequence by reacting again with another free radical [15]. (See Reactions B)

$$
\begin{aligned}
& \text { Reactions A: } \quad \mathrm{ROO}+\mathrm{AH}->\mathrm{ROOH}+\mathrm{A}^{-} \\
& \mathrm{RO}+\mathrm{AH}->\mathrm{ROH}+\mathrm{A}^{\cdot} \\
& \text { Reactions B: } \quad \mathrm{ROO}+\mathrm{A}^{-}->\text {ROOA } \\
& \mathrm{RO}+\mathrm{A}^{*}->\mathrm{ROA}[16]
\end{aligned}
$$

The ability of a compound to act as an antioxidant depends highly on its chemical structure. In the case of polyphenolic compounds, the group, flavonoids, contains the highest ability to act as an antioxidant due to its natural structure. Containing such compounds as an $o$-diphenolic group, a 2-3 double bond conjugated with the 4-oxo function, and ideally placed hydroxyl groups on the rings, the flavanoid is able to give off hydrogen and become a radical without losing much stability [17-19].

As antioxidants, proanthocyanidins have high oxygen radical scavenging capacity in human body. Foods rich in proanthocyanidins have been linked to a number of health benefits ranging from cardiovascular health, cancer prevention, and anti-aging. They are also shown to protect body from environmental stresses such as smoking and pollution [20].

\section{METHODS OF SEPARATION}

\subsection{Various Separation Methods}

The method used to separate proanthocyanidins from grape seeds is just as important as the benefit that they provide to the person consuming them. Without proper knowledge of extraction techniques, one cannot characterize these compounds properly. Due to their variations in size and structure, most current separation methods are designed to not only extract one particular type of proanthocyanidin, but also extract as many different types as the methods can. However, with the complex nature of some proanthocyanidins, most methods have been shown to prove to be effective on the lower molecular weight polyphenolic compound.

The method which has been utilized most often in separation of proanthocyanidins from grape seeds is liquid/liquid extraction using various polar solvents, sometimes with the help of ultrasound, microwave, enzyme, and mechanical treatment of seeds [21, 22]. Subcritical water extractions of proanthocyanidins from grade seeds have also been reported $[23,24]$ and supercritical $\mathrm{CO}_{2}$ technique was used to extract grape seed oil that contains proanthocyanidins [25]. While the main liquid in liquid/liquid extraction does vary, the liquid/liquid extraction appears to usually utilize water containing polar solvent; the amount varies depending on the asso- 
ciative liquid in the system. Exclusive as the extraction can be, characterization of these compounds appears to be highly dependent on exactly what the experimenter is trying to achieve with the experiment. If he or she intends to generate significant amounts of test compounds, which then can be used for bioavailability and bioactivity studies, and analytical purposes, then a more elaborate preparative isolation of phenolic compounds by using chromatography is called for. If he or she intends to obtain maximum amount of phenolic compounds such as proanthocyanidins from grade seeds, a large throughput process may be used and HPLC is then employed to investigate the presence of several categories of phenolic compounds. To complicate the matter further, the phenolic compounds are very unevenly distributed in grape seeds with up to $90 \%$ phenolic compounds found in seed coat [26]. Thus, preparatory steps before liquid/liquid extraction are very important in obtaining maximum yield and minimum impurity.

\subsection{Liquid/Liquid Extraction with Ethyl Acetate}

Liquid/liquid extraction is the main method of isolating proanthocyanidins from grape seeds and other materials that contain this type of compounds. Ethyl acetate and water is the most commonly used liquid/liquid solvent system of this method [26, 27]. A typical procedure using this solvent mixture involves a 24 hour wait, decantation of the extract, drying, and mixing with a precipitating agent, such as petroleum ether, which allows the solid to be filtered out late on [2830]. Other extraction methods that use methanol, ethanol, and acetone (all with water) have proven to be effective, but not as effective as ethyl acetate and water for extracting proanthocyanidins [31]. The higher the number of hydroxyl groups found on the proanthocyanidin, the easier it can be extracted using this type of liquid/liquid extraction.

The initial form of grape seed is important to take into consideration for extracting proanthocyandins. Most extractions in the studies began with whole grapes, handpicked from a specific location with the full skin intact. The skin and flesh are then hand removed and the seeds are thus separated [30, 32]. Upon drying, the seeds are ready for extraction and can be stored under standard freezing conditions until use. There is no information found on whether or not freezing would damage the integrity of the seeds themselves or the proanthocyanidins they contain. Proanthocyanidins can be extracted from the seeds as a whole, or the seeds may be ground and then extracted. The general observation from several studies has shown that grinding grape seeds would not result in an increase in amount of proanthocyanidins extracted, but rather a reduction in the time of the extraction $[28,31]$. However, these accounts have also noted that grinding leads to more impurities being produced in the extraction.

Several studies have been performed to identify the optimal conditions of extraction using ethyl acetate and water. While it appears to be possible to extract proanthocyanidins from grape seeds only using ethyl acetate, it also appears impractical due to its long rate of reaction. The ethyl acetate alone cannot permeate through the grape seed matrices thus allowing the release of the proanthocyanidins from the seeds. Combining the ethyl acetate with water directly affects the reaction rate because the water helps to increase permeability [29].

Water added to the system shows a direct relationship with an increase in yield and also in reaction rate of the extraction of proanthocyanidins. Based on the studies performed, extraction of polyphenolic compounds found in grape seeds is optimal when the extraction system is saturated with water. This optimum is found to be $10 \%$ water content in the system of ethyl acetate/water (90:10), assuming that the water content in the saturated grape seeds is found to be between $33-35 \%$ and that ethyl acetate is saturated with water between 3.3-3.5\%. When water concentration in the extraction system is below $10 \%$, it was found that since the grape seeds are not saturated, optimum selectivity and yield could not be reached. This observation also holds true for extraction systems which contain more than $10 \%$ water. These higher water concentration extraction systems experienced a decrease in selectivity as well; however they did experience a small increase in yield of proanthocyanidins from the grape seeds $[28,29]$.

Extraction time is also an important factor in extraction of proanthocyandins from grape seeds. The optimal reaction time from the extraction studies was shown to be 24 hours for extracting polyphenols from grape seeds. However, although the 24-hour time frame does result in the maximum amount of proanthocyanidins being extracted from grape seeds, the question remains: is this really necessary? It has been shown that extraction increases rapidly up to 15 hours and then plateau out after that. While maximum amounts can be obtained after 15 hours, in most cases, 15 hours is enough time to collect sizable amounts of proanthocyanidins from grape seeds [28, 29].

\subsection{Separation with Other Solvents and Techniques}

Solvents other than ethyl acetate can be used to extract proanthocyanidins from plant materials that contain these compounds. While ethyl acetate is ideal to use in this type of extraction-characterization work, in reality, various other solvents that vary in polarity are available to use in liquid/liquid extraction of proanthocyanidins. This is particularly true in extracting proanthocyanidins for functional food and nutraceutical uses where ethyl acetate/water system or methanol/water system may not be as appropriate as ethanol/water extraction system or subcritical water system. There are not many documented studies in English language to be found on this topic, however, several reports have appeared in various journals of different languages [23, 24, 33]. Countercurrent chromatography and solid phase extraction can also be used with abovementioned solvents to improve yield and efficiency. All liquid/liquid extraction techniques can be further assisted with microwave, ultrasound, and thermo-mechanical (including extrusion) and enzymatic pretreatment of grape seeds prior to extraction [21-24]. Concentration of phenolic rich grape seed extracts can be made by vacuum evaporation and ultrafiltration/nanofiltration [34]. Nawaz et al. [35] described a solvent extraction method using $50 \%$ ethanol and $50 \%$ water as solvent to extract polyphenols from grape seeds. Ultrafiltration membrane system with average pore size of $0.22 \mu \mathrm{m}$ was utilized to concentrate extracts. Since the process does not involve any toxic sol- 
vent, this approach can be adopted for the use in the food and nutritional supplement industries. For analytical purposes that require purified compounds, purification can be achieved through preparative chromatography. Based on the current literature search, it appears that ethyl acetate/water system is the standard for extraction of proanthocyanidins from grape seeds for analytical purposes. Other methods should not be considered nearly as widely used for extracting analytic quality of proanthocyanidins.

Other methods of liquid/liquid extraction include using methanol, acetone, ethanol, and water in various combinations and different proportions. The number of combinations that can result from is huge. Therefore no comparisons can be made, due to the diversity of amounts used of each extractant, plant material used, analytical methods used afterwards for characterization, and amount of substances that are not proanthocyanidin extracted in addition to the desired product. Unlike the ethyl acetate and water liquid/liquid extraction system, there is no data that gives any sort of credence that one extractant is better than another, except for Dumon's paper in 1990 [31]. He found among the above liquid/liquid extraction systems, excluding ethyl acetate, acetone/water produces a better desired result of extraction of proanthocyanidins than the other liquid/ liquid systems [31]. This section will therefore not try to establish a standard of extraction of proanthocyanidins. Rather it is just for overview of what else may be used to extract proanthocyanidins from various plant materials, not limited to grape seeds.

Methanol can be used as an extract agent for proanthocyanidins. It appears to be used in conjunction with the method of fractionation to recover proanthocyanidins from various plant materials. This method is also usually used with high-performance liquid chromatography (HPLC). Methanol can be used as the only extracting agent or in conjunction with other agents. Most often than not, it was used either alone or with water in the extraction systems [36-40]. There does not appear to be an established standard amount of water used with methanol as in the case with ethyl alcohol; nor does there appear to be an established standard time that is associated with this method.

Methanol can also be used in conjunction with other extractants which are not ethanol, acetate, or water in different methods of proanthocyanidin separation. One paper suggests that methanol and chloroform may be used in conjunction with glass power to extract the polyphenolic compounds based on their degree of polymerization [41]. Other papers suggest a different method that uses methanol and chloroform to complete a series of successive precipitations without the need for glass powder. This method chooses to use glass filters only instead [30, 42].

Acetone can also be considered a common extractant [43]. Considered the best versus methanol and ethanol when combined with water [31], this extractant is commonly used when it is used in series with another extractant. Typically, that extractant is methanol, ethanol, ethyl acetate, or water in some combinations [30, 44-46].

\section{CHARACTERIZATION OF PROANTHOCYNIDINS}

\subsection{Overview of Advanced Analytical Techniques}

The identification and characterization of proanthocyanidins can be accomplished through a number of methods. The nature of a scientific study usually dictates which method is going to be used: whether the experiment is used to identify a new compound, develop a new method to replace a current standard, or simply to show that the healthful polyphenolic compounds were indeed extracted from the method used.

The five most common advanced analytical techniques used for proanthocyanidin characterization are: high performance liquid chromatography (HPLC), both carbon and hydrogen nuclear magnetic resonance (NMR), mass spectroscopy (MS), infrared spectroscopy (IR), and gas chromatography (GC). Of these methods, HPLC is the most widely and commonly used to identify the presence of proanthocyanidin compounds within extracted samples using ultra-violet (UV) detection. The rapidity of the experiment and the ease of identification of compound amount eluted probably is a key factor in the polarity of this method. NMR, MS, and IR are also common methods to demonstrate the presence of proanthocyanidins. However, the usefulness of these techniques lies in their ability to characterize new structures and higher molecular weight proanthocyanidins. So while HPLC is used mainly for identifying presence and amount of proanthocyanidins, the other techniques are mainly used for identifying the actual molecules. Again, this section will focus on, but not be limited to, the proanthocyanidins found in grape seeds. It is the belief of the authors that limiting this section to only proanthocyanidins from grape seeds would not be able to gather a full view of all methods/techniques used to characterize this healthful polyphenolic compound.

\subsection{High Performance Liquid Chromatography}

HPLC is based on the idea of non-covalent interactions between compounds. A column is used as a stationary phase in which a solvent, also referred to as the mobile phase, is continuously applied to the column itself. The sample, in this case the liquid that contains proanthocyanidin compounds, would be injected into the column with the mobile phase at a given time. The sample will interact with both the mobile and stationary phases through non-covalent interactions based on the compound that is present. Presumably, the compound that has more ionic attribute will react more strongly than a compound that has no ionic attribute at all. The interaction of the compound with the phases is the basis for the eluting of the compound from the column. The more ionic the compound, the longer it will take to elute due to the increased retention time of the compound. A detector can be attached at the end of the column to allow for the identification of different compound groups and amounts based on this mobile phase/compound interaction. Often this detector is an MS or a UV detector set at a wavelength of $280 \mathrm{~nm}$.

The problem with this method of compound identification is the lack of ability to correlate data between various experiments. The establishment of the effectiveness and the degree of accuracy of this method of proanthocyanidin identification is well known for both reverse phase and normal phase HPLC for grape seeds, grapes, and other proanthocyanidin containing materials [30, 36, 47-49]. How- 
ever, there does not appear to be a standard mobile phase or stationary phase used with this method of component identification/characterization. Without a standard mobile and stationary phase, data cannot be correlated between various experiments due to the varying retention times of the compounds themselves with the phases.

What appears to have become the norm in this method is to simply rely on a previous experimenter's decision of mobile and stationary phases for the development of a current HPLC. The results of the experiment are then compared to commercial standards of the known compounds which are run through the same column using the same method as the unknown compounds to be identified.

Saucier's experiment [30] adapts its method of normal HPLC analysis from Matthews et al. 1997 [50]. Using a flow rate of $1 \mathrm{~mL} / \mathrm{min}$, the column was kept at a temperature of $30^{\circ} \mathrm{C}$ with an injection volume of $20 \mu \mathrm{L}$ and a UV detector at wavelength of $280 \mathrm{~nm}$. Solvent A was water with $5 \%$ acetic acid. Solvent B was $\mathrm{MeOH}$ with $5 \%$ acetic acid. The gradient was as follows: $30 \%$ to $100 \%$ solvent $\mathrm{B}$ in 35 minutes, $100 \%$ solvent B during 5 minutes, and finally $100 \%$ to $30 \%$ solvent B in 5 minutes. The injected material had previously undergone thiolysis and extraction from the grape seeds via acetone and ethyl acetate.

Pekia's 1998 [28] study based their study's reverse phase HPLC analysis off of a paper written in 1995. The proanthocyanidins were isolated using methanol and vanillin reagent. Using $10 \%$ acetic acid as solvent A and water as solvent $\mathrm{B}$, these solvents used a standard linear gradient, as demonstrated in their paper, at $32^{\circ} \mathrm{C}$. A variable wavelength detector of UV light set at 280nm again was used as a detector in this HPLC experiment. Flow rate was not given [28, 51].

Porter isolated cranberry proanthocyanidins via a currently non-established method of HPLC gradient. Using a six fraction gradient as the mobile phase; water (fraction 1), water/ethanol (1:1) (fraction 2), ethanol (fraction 3), ethanol/ methanol (1:1) (fraction 4), methanol (fraction 5), and aqueous acetone (fraction 6); proanthocyanidins were eluted through the column at a rate of $1 \mathrm{~mL} / \mathrm{min}$. The stationary phase was $70 \mathrm{~g}$ of Sephadex LH-20 hand packed into the column. The peaks were monitored at 280, 320, 360, and $520 \mathrm{~nm}$ through a diode array collector demonstrated that proanthocyanidins were collected in fractions 4-6 with peaks at 280nm [52].

As it can be seen through the last three examples, the conditions for each experiment were different. Standardization between the resultant values is not possible with this diversity of solvent used on the varying columns. What is consistent though is the ability to detect proanthocyanidins by UV detectors at $280 \mathrm{~nm}$. Though HPLC is the most common method of characterizing proanthocyanidin content within a material when used with another method of identification, such as MS or UV detectors, HPLC alone is not capable of proanthocyanidin characterization.

\subsection{Mass Spectroscopy, NMR \& Near Infrared Spectros- copy}

Most other advanced analytical techniques are solely used as a means of compound identification and not as a method of isolation or quantification. These methods are commonly used when the investigator is trying to determine molecular structures of specific healthful compounds within a given product, e.g. grape seeds, cranberries, or any other proanthocyanidin containing compound. The identification of new proanthocyanidins is also achieved via these more compound specific techniques.

Mass spectroscopy used mainly for composition determination. This method can be explained by examining the experiments performed by Kendil et al. [53]. The cranberry fruit contains multiple polyphenolics within a group that inhibits ornithine decarboxylase activity, induced via a specific pathway. Using mass spectroscopy techniques, coupled with other identification methods, the proanthocyanidins responsible for this were identified and can be further studied to help understand this mechanism [53].

Both hydrogen and carbon NMR are also used for identification purposes. Several papers demonstrated the use of this advanced analytical technique for specific compound identification within a group of molecules. Stevens et al. and Giess et al. both use this method to determine chemical structures within a group of proanthocyanidins. Both use a combination of advanced techniques to achieve this end [5355]. While Cui et al. uses a slightly different method of 2D NMR to determine molecular structure of the unknown compounds [56]. It is impossible to use any version of NMR without some sort of prior isolation technique of the compound one is trying to identify.

Near infrared spectroscopy (NIRS) is a rapid nondestructive method that is capable of detecting and measuring a complex number of general and specific components in both solid and liquid samples, including dissolved solids, acid concentrations, density, $\mathrm{pH}$, microbial contamination, and percent content of oil, carbohydrate, protein, and moisture. NIRS is also capable of accurately determining the concentrations of specific chemical components. The advent of rapid computer programs that utilize complex mathematical calculations, including Fourier transformation, has allowed for the computation of relationships between variation of multiple parameters and corresponding NIR spectra. In addition, the long path lengths in NIRS allow for sampling through glass and plastic, making NIRS an even more efficient measuring process. When coordinated with proven chemical and physical analytical methods, the analytical results can be translated into reliable NIR spectrometric calibrations. This can potentially measure various phytochemical components in small amounts of plant tissues. FerrerGallego et al. [57] explored the potential of NIRS in determining contents of flavanols based on the recorded infrared spectra of grape seed compounds. Their interest in the study was mainly to find a way of distinguishing different grape varietals from different regions by comparing the flavanols contents of samples. This is useful in finding counterfeits and mislabeling of wine and grape products. How much this technique can be used in characterizing proanthocyanidins in grape seeds is an open question since compounds of proanthocyanidins are so diverse and very difficult to be purified and concentrated to be use as standards for NIRS 
calibration, a necessary step to use NIRS to determine proanthocyanidins in grape seeds.

\section{CONCLUSIONS}

Proanthocyanidins are a class of biologically active flavonoids found throughout the plant kingdom, and are one of the most potent antioxidants in nature. Recent studies in animals, as well as in some human studies, have shown that grape seed proanthocyanidin extracts possess a broad spectrum of biological, pharmacological and chemoprotective properties against free radicals and oxidative stress. Some grape seed extracts have been reported to promote collagen formation, enhance cardiovascular health, improve microcirculation, prevent many of the effects related to premature aging, inhibit growth and formation of certain types of cancer, and protect cells against drug, chemical and environmental pollutants toxicity.

The need to extract maximum amount of proanthocyandins from grape seeds for food and nutraceutical uses is the driving force of the current studies of proanthocyanidin extraction. Then search for economically and ecologically feasible extraction technologies continues unabated. There are several methods available for extracting proanthocyanidins from grape seeds using organic solvent and combination of water and polar solvents. Novel extraction and concentration methods continue to develop to reduce costs and improve quality of proanthocyanidins from grape seeds. Several grape seed extracts have been marketed in a number of countries.

Characterization of proanthocyandins using wet chemistry and instruments continues to evolve and more research is required to overcome the current technical barriers and improve the accuracy of analysis. Collaborations among researchers and analytical instrument manufacturers are duly needed.

\section{CONFLICT OF INTEREST}

None declared.

\section{ACKNOWLEDGEMENTS}

None declared.

\section{REFERENCES}

[1] Harborne JB. Methods in plant biochemistry I: Plant phenolics. New York, London: Academic Press 1990.

[2] Nakamura Y, Tsuji S, Tonogai Y. Analysis of proanthocyanidins in grape seed extracts, health foods and grape seed oils. J Health Sci 2003; 49: 45-54.

[3] Fine AM. Oligomeric proanthocyanidin complexes: history, structure, and phytopharmaceutical applications. Altern Med Rev 2000; 5: $144-51$.

[4] Hertog MGL, Sweetnam PM, Fehily AM, Elwood PC, Kromhout D. Antioxidant flavonols and ischaemic heart disease in Welsh populations of men. The caerphilly study. Am J Clin Nutr 1997; 65: 1489-94.

[5] Hertog, MGL, van Poppel G, Verhoeven D. Potentially anticarcinogenic secondary metabolites from fruits and vegetables. In: Tomas-Barberan, FA, Robins, RJ, Eds. Phytochemistry of fruits and vegetables. Oxford: Clarendon Press 1997; pp.313-29.

[6] Harborne JB. The flavonoids: advances in research since 1986. London: Chapman and Hall 1993.
[7] Harborne JB, Mabry TJ. The flavanoids: advances in research. New York: Chapman and Hall 1982.

[8] Hertog MGL, Feskens EJM, Hollman PCH, et al. Dietary antioxidant flavonoids and risk of coronary heart disease: the Zutphen elderly study. Lancet 1993; 342: 1007-11.

[9] Hertog MGL, Kromhout D, Aravanis C, et al. Flavonoid intake and long-term risk of coronary heart disease and cancer in the seven countries study. Arch Intern Med 1995; 155: 381-6.

[10] Bagchi D, Bagchi M, Stohs SJ, et al. Free radicals and grape seed proanthocyanidin extract: importance in health and disease prevention. Toxicology 2000; 148:187-97.

[11] Plumb GW, De Pascual-Teresa S, Santos- Buelga C, et al Antioxidant properties of catechins and proanthocyanidins: effect of polymerisation, galloylation, and glycosylation. Free Radic Res 1998; 29: 351-8.

[12] Packer L, Rimbach G, Virgili F. Antioxidant activity and biological properties of a procyanidin-rich extract from pine (Pinus maritima) bark, pycnogel. Free Radic Biol Med 1999; 27: 704-24.

[13] Renaud S, DeLorgeril M. Wine, alcohol, platelets, and the French paradox for coronary heart disease. Lancet 1992; 339: 1523-6.

[14] Fuhrman B, Lavy A, Aviram M. Consumption of red wine with meats reduces the susceptibility of human plasma and LDL to lipid peroxidation. Am J Clin Nutr 1995: 61: 549-54.

[15] Shahidi F, Wanasundara PKJPD. Phenolic antioxidants. Crit Rev Food Sci Nutr 1992; 32: 67-103.

[16] Bruice PY. Organic Chemistry. $3^{\text {rd }}$ ed. New Jersey: Prentice Hall 2001.

[17] Manch C, Regerat F,Texier O, et al. Bioavailability, metabolism, and physiological impact of 4-oxo-flavonoids. Nutr Res 1996; 16: 517-44.

[18] Bors W, Heller W, Michel C, Saran M. Flavonoids as antioxidants: determination of radical-scavenging efficiencies. Methods Enzymol 1990; 186: 343-55.

[19] Ratty AK, Das NP. Effects of flavonoids on nonenzymic lipid peroxidation: structure activity relationship. Biochem Med Metab Biol 1988; 39: 69-79.

[20] Cos P, De Bruyne T, Hermans N, Apers S, Berghe D, Vietinck A. Proanthocyanidins in health care: current and new trends. Curr Med Chem 2004; 11(10): 1345-259.

[21] Li C, Wang W, Zheng Y, Fan D, Xie X. Extraction of proanthocyanidins from grape seeds with ultrasonic wave. Zhongguo Shipin Tianjin (Chinese Food Products Tainjin) 2010; 2: 68-71. (In Chinese).

[22] Zhang L, Sun X, Wang S, Tan T. Microwave extraction of proanthocyanidins from grape seeds. Zhong Cao Yao (Chinese Traditional and Herbal Medicines) 2007; 38(12): 1808-11. (In Chinese).

[23] Garcia-Marino M, Rivas-Gonzalo J, Ibanez E, Garcia-Moreno C. Recovery of catechins and proanthocyanidins from winery byproducts using subcritical water extraction. Anal Chim Acta 2006; 563(1-2): 44-50.

[24] Li C, Zheng Y, Wang W, Wang N, Yang R. Optimization of subcritical water extraction of proanthocyanidins from grape seeds by response surface methodology. Shipin Kexue (Food Science) 2010; 31(12): 6-10. (In Chinese).

[25] Wang J, Yang J, Zhao P. Optimization of supercritical $\mathrm{CO}_{2}$ extraction of grape seed oil containing proanthocyanidins by uniform design. Zhongguo Youzhi (Chinese Oils Fats) 2009; 34(7): 8-11. (In Chinese).

[26] Xu C, Zhang Y, Wang J, Lu J. Extraction, distribution and characterization of phenolic compounds and oils in grape seeds. Food Chem 2010; 122(3): 688-94.

[27] Wen G, Li L, Sui X. Extraction of oligomeric proanthocyanidins from grape seeds. Zhongguo Niangzao (China Brewing) 2010; 1: 111-3. (In Chinese).

[28] Pekia B, Kovaa V, Alonsod E, Revillad E. Study of the extraction of proanthocyanidins from grape seeds. Food Chem 1998; 61: 2016.

[29] Wei F, Han J, Zhang L, Yang H, Wang Z. Study on technology of extracting oligoprocyanidins from grape seeds. Xian Dai Hua Gong (Modern Chemical Industry) 2001; 21 (4): 27-9. (In Chinese)

[30] Saucier C, Mirabel M, Daviaud F, Longieras A, Glories Y. Rapid fractionation of grape seed proanthocyanidins. J Agric Food Chem 2001; 49: 5732-5. 
[31] Dumon MC. Recherches analytiques sur les picnogenols. These pour le Doctorat d'Etat des Sciences pharmaceutique. Bordeaux, France: Universite de Bordeaux II 1990.

[32] Peng Z, Hayasaka Y, Iland PG, Sefton M, Hoj P, Waters E. Quantitative analysis of polymeric procyanidins (tannins) from grape (Vitis vinifera) seeds by reverse phase high-performance liquid chromatography. J Agric Food Chem 2001; 49: 26-31.

[33] Wang W, Li C, Ling L. Abstraction and separation of proanthocyanidins from grape seeds. Zhongguo Shipin (Chinese Food Products) 2010; 3: 84-9. (In Chinese).

[34] Zhang L, Sun X, Wang S, Tan T. Microwave extraction of proanthocyanidins from grape seeds. Zhong Cao Yao (Chinese Traditional and Herbal Medicines) 2007; 38(12): 1808-11. (In Chinese).

[35] Nawaz H, Shi J, Mittal GS, Kakuda Y. Extraction of polyphenols from grape seeds and concentration by ultrafiltration. Sep Purif Technol 2006; 48: 176-81.

[36] Fuleki T, Ricardo da Silva JM. Catechin and procyanidin composition of seeds from grape cultivars grown in Ontario. J Agric Food Chem 1997; 45:1156-60.

[37] Nitao JK, Birr BA, Nair MG, Herms DA, Mattson WJ. Rapid quantification of proanthocyanidins (condensed tannins) with a continuous flow analyzer. J Agric Food Chem 2001; 49: 2207-14.

[38] Guyot S, Doco T, Souquet JM, Moutounet M, Drilleau JF. Characterization of highly polymerized procyanidins in cider apple (Malus sylvestris var. Kermerrien) skin and pulp. Phytochemistry 1997; 44(2):351-7.

[39] Fitzpatrick DF, Fleming RC, Bing B, Maggi DA, O’Malley RM. Isolation and characterization of endothelium-dependent vasorelaxing compounds from grape seeds. . J Agric Food Chem 2000; 48: 6384-90.

[40] Stafford HA, Cheng TY. The procyanidins of Douglas fir seedlings, callus, and cell suspension cultures derived from cotyledons. Phytochemistry 1980; 19: 131-5.

[41] Labarbe B, Cheynier V, Bossaud F, Souquet JM, Moutounet M. Quantitative fractionation of grape proanthocyanidins according to their degree of polymerization. J Agric Food Chem 1999; 47: 271923.

[42] Glories Y. Recherches sur la matiere colorante des vins rouges. Ph.D. Dissertation. France: University Victor Segalen Bordeaux 1978.

[43] Hernandez-Jimenez A, Gome-Plaza E, Martinez-Cutillas A, Kennedy J. Grape skin and seed proanthocyanidins from Monastrell $\times$ Syrah grapes. J Agric Food Chem 2009; 57: 10798803.

[44] Naczk M, Amarowicz R, Zadernowski R, Shahidi F. Protein precipitating capacity of condensed tannins of beach pea, canola hulls, evening primrose, and Fava bean. Food Chem 2001; 73: 467-71.

[45] Adamson GE, Lazarus SA, Mitchell AE, et al. HPLC method for the quantification of procyanidins in cocoa and chocolate samples and correlation to total antioxidant capacity. J Agric Food Chem 1999; 47: 4184-8.

[46] Nonaka GI, Miwa N, Nishioka I. Stilbene glycoside gallates and proanthocyanidins from Polygonum multiflorum. Phytochemistry 1982; 21: 429-32.

[47] Kovac V, Alonson E, Revilla E. The effect of adding supplementary quantities of seeds during fermentation on the phenolic compositions of wines. Am J Enol Vitic 1995; 46: 363-7.

[48] Oszamianski J, Sapis JC. Fractionation and identification of some low molecular weight grape seeds phenolics. J Agric Food Chem 1989: 37: 1293-7.

[49] Rigaud J, Escribano-Bailon MY, Prieur C, Souquet JM, Cheynier V. Normal-phase high performance liquid chromatographic separation of proanthocyanidins from cacao beans and grape seeds. J Chromatogr 1993; 654: 255-60.

[50] Matthews S, Mila I, Scalbert A, et al. Method for estimation of proanthocyanidins based on their acid depolymerization in the presence of nucleophiles. J Agric Food Chem 1997; 45:1195-201.

[51] Revilla E, Escalona JM, Alonso E, Kova V. The phenolic composition of table grapes. In: Charlambous G, Ed. Food flavors: generation, analysis, and process influence. Amsterdam: Elsevier Sci 1995; pp. 1579-96.

[52] Rigaud J, Escribano-Bailon MY, Prieur C, Souquet JM, Cheynier V. Normal-phase high performance liquid chromatographic separation of proanthocyanidins from cacao beans and grape seeds. J Chromatogr 1993; 654: 255-60.

[53] Kandil FE, Smith ML, Rogers RB, et al. Composition of a chemopreventive proanthocyanidin-rich fraction from cranberry fruits responsible for the inhibition of 12-O-tetradecanoyl phorbol13-acetate (TPA)-induced ornithine decarboxylase (ODC) activity. J Agric Food Chem 2002; 50:1063-9.

[54] Stevens JF, Miranda CL, Wolthers KR, Schimerlik M, Deinzer ML, Buhler DR. Identification and in vitro biological activities of hop proanthocyanidins: Inhibition of nNOS activity and scavenging of reactive nitrogen species. J Agric Food Chem 2002; 50: 343543.

[55] Geiss F, Heinrich M, Hunkler D, Rimpler H. Proanthocyanidins with (+)-Epicatechin units from Byrsonima crassifolia bark. Phytochemistry 1995; 39: 635-43.

[56] Cui CB, Tezuka Y, Kikuchi T, Nakano H, Tamaoki T, Park JH. Constituents of a fern, Davallia mariesii Moore. II. Identification and $1 \mathrm{H}$ - and 13C-nuclear magnetic resonance spectra of procyanidin B-5, epicatechin-(4 beta----8)-epicatechin-(4 beta---6)-epicatechin, and epicatechin-(4 beta----6)-epicatechin-(4 beta---8)-epicatechin-(4 beta----6)-epicatechin. Chem Pharm Bull 1992; 40 (4): 889-98.

[57] Ferrer-Gallego R, Hernández-Hierro JM, Rivas-Gonzalo JC, Escribano-Bailón MT. Feasibility study on the use of near infrared spectroscopy to determine flavanols in grape seeds. Talanta 2010; 82(5):1778-83. 